\title{
Optimizations of $\mathrm{Pt} / \mathrm{SiC}$ and $\mathrm{W} / \mathrm{Si}$ multilayers for the Nuclear Spectroscopic Telescope Array.
}

\author{
Kristin K. Madsen, Fiona A. Harrison and Peter H. Mao ${ }^{a}$. Finn E. Christensen, Carsten P. \\ Jensen and Nicolai Brejnholt ${ }^{b}$. Jason Koglin ${ }^{c}$. Michael J. Pivovaroff ${ }^{d}$. \\ ${ }^{a}$ California Institute of Technology, Pasadena, United States \\ ${ }^{b}$ National Space Institute, DTU-Space, Copenhagen, Denmark \\ ${ }^{\circ}$ Columbia University, New York, United States \\ ${ }^{d}$ Lawrence Livermore National Laboratory, Livermore, United States
}

\begin{abstract}
The Nuclear Spectroscopic Telescope Array, NuSTAR, is a NASA funded Small Explorer Mission, SMEX, scheduled for launch in mid 2011. The spacecraft will fly two co-aligned conical approximation Wolter-I optics with a focal length of 10 meters. The mirrors will be deposited with $\mathrm{Pt} / \mathrm{SiC}$ and $\mathrm{W} / \mathrm{Si}$ multilayers to provide a broad band reflectivity from $6 \mathrm{keV}$ up to $78.4 \mathrm{keV}$. To optimize the mirror coating we use a Figure of Merit procedure developed for gazing incidence optics, which averages the effective area over the energy range, and combines an cnergy weighting function with an angular wcighting function to control the shape of the desired effective area. The NUSTAR multilayers are depth graded with a power-law, $d_{i}=a /(b+i)^{c}$, and we optimize over the total number of bi-layers, $\mathrm{N}, c$, and the maximum bi-layer thickness, $d_{\max }$. The result is a 10 mirror group design optimized for a flat even energy response both on and off-axis.
\end{abstract}

\section{INTRODUCTION}

Current and past x-ray space missions such as Chandra, ${ }^{1}$ XMM-Newton ${ }^{2}$ and Einstein, ${ }^{3}$ have used Wolter- ${ }^{4}$ mirrors coated with a single layer of gold or iridium to reflect and focus $\mathrm{x}$-rays. To achieve a high response in their respective energy bands, they employed incidence angles, $\theta_{i n c}$, less than the critical angle, $\theta_{c}$, below which a ray is totally externally reflected. The critical angle is energy and material dependent and given by

$$
\theta_{c}=\sqrt{2 \delta}=\frac{\sqrt{4 \pi \rho r_{0}}}{k}
$$

where $\rho$ is the material density, $k$ the wave number and $r_{0}$ the Thompson scattering length. ${ }^{5}$ This translates into focal length and collecting area, as a longer focal length for a fixed size optic will enable it to reflect higher energies. For missions like Chandra and XMM-Newton the focal length was limited by practical constraints in space for a solid structure, and so beyond $\sim 10 \mathrm{keV}$ neither missions have any reflection capabilities, as the critical angles required to reflect beyond $\sim 10 \mathrm{keV}$ are smaller than the smallest $\theta_{\text {inc }}$ of the telescopes.

A solution to impractically long focal lengths and the restriction of the critical angle is multilayer coatings. A multilayer is a stack of thin films of two different materials, stacked alternately one top of the other. Enhanced reflectivity beyond the critical angle is achieved from a multilayer stack through two effects; the Bragg law and the density contrast at the interface of reflection. The stack acts as a periodic lattice and the Bragg condition will create constructive interference according to $\lambda m=2 d \sin \theta_{i}$, where $\mathrm{d}$ is the lattice spacing, $\theta_{i}$ the incidence angle and $m$ the order of reflection. ${ }^{5}$ The probability of reflection at the interface is proportional to the density contrast of the two materials, and multilayers are composed of a high density and low density material, such as $\mathrm{W} / \mathrm{Si}$ and $\mathrm{Pt} / \mathrm{SiC}$.

NuSTAR uses multilayers to push past the $\sim 10 \mathrm{keV}$ limit, extending the bandpass all the way up to 78.4 $\mathrm{keV}$ where the Pt K-edge absorption cuts down the reflectivity, while still maintaining a reasonable focal length of 10 meters. Although the multilayer parameter space is to some degree constrained by fabrication, the space remains large, and here we will focus on efforts to find the optimum multilayer design for the NuSTAR mission.

\footnotetext{
Optics for EUV, X-Ray, and Gamma-Ray Astronomy IV, edited by Stephen L. O'Dell, Giovanni Pareschi, Proc. of SPIE Vol. 7437, 743716-@ 2009 SPIE CCC code: 0277-786X/09/\$18 - doi: 10.1117/12.826669
} 


\section{MISSION OVERVIEW}

The Nuclear Spectroscopic Array, NuSTAR, ${ }^{6}$ is a NASA funded and scheduled for launch in mid 2011. The spacecraft will be lamnched into an equatorial 6 deg orbit, and during its two year mission lifetime will be studying galactic and extra-galactic high energy processes such as supernovae remnant and super massive black holes.

The spacecraft will fly two co-aligned conical approximation Wolter-I optics with a focal length of 10 meters. The optics are segmented in the azimuth into twelve sections, and length wise into 2 sections, upper conical section and lower conical section. There will be a total of 130 shells and the mirrors will be deposited with $\mathrm{Pt} / \mathrm{SiC}$ and W/Si multilayers to provide reflectivity up to $78.4 \mathrm{keV}$. Using technology developed for Constallation-X, thin borosilicate mirror substrates are slumped into cylindrical shape at Goddard Space Flight Center. The substrates have a Half Power Diameter, HPD, of 20 - 40 arc-seconds, mainly limited by mid spatial figure error from length scales from 2 to $20 \mathrm{~mm}$, which when mounted are expected to yield an average IIPD of 40 arc-seconds. The mirrors will be coated with multilayers at the National Space Institute, DTU-space, Denmark, where in the past the balloon mission HEFT has had its optics coated. ${ }^{7}$ The Pt/SiC coatings are annealed to 250 degC for 2 hours which reduces the residual stress to below $50 \mathrm{Mpa}$, minimizing any figure distortions due to coating induces stress. The optics will be assembled at Columbia, where the segments are glued with epoxy onto precisely machined graphite spacers.

NuSTAR will have two focal planes connected to the optics by a 10 meter extendable mast. Each is equipped with a square array of four CdZnTe hybrid detectors covering a Field of View of 13.4 arc-minutes, housed in an active NaI shield. One hybrid has $32 \times 32$ pixels connected to custom designed ASIC read-out chip developed at California Institute of Technology.

\section{MULTILAYER}

Multilayers have long been in use for UV lithography, neutrons beam lines and in laboratory x-ray systems to focus and shape the beams, and only in recent years have these principles been applied to astrophysical optics:

A multilayer is a stack of thinly deposited films of alternating material, where one set of the high- $\mathrm{Z}$ and low- $\mathrm{Z}$ film is a bi-layer. The thickness of the individual films will determine the shape of the spectrum. For example a constant thickness of the bi-layer will result in a spectrum with a good response at a few monochromatic energies, at the location of the Bragg peak, but otherwise have a poor broad band spectral response. Far more convenient is a power-law graded stack, ${ }^{8}$ which through a varying bi-layer thickness ensures that the Bragg peaks are shifted through the spectrum providing a broad band response. The bi-layer thickness of the power-law stack is defined by

$$
d_{i}=\frac{a}{(b+i)^{c}} \quad i=1, N
$$

where $N$ is the number of bi-layers in the stack. The bi-layer $\mathrm{i}=1$ is the top and thickest bi-layer. The fraction of the high density material to the thickness of the bi-layer is given by $\Gamma=d_{\text {highz }} / d$, and once $c$ is chosen then the maximum and minimum d-spacing, $d_{\text {max }}, d_{\min }$, determines parameters $a$ and $b$. The power law index $c$ controls the relative shifts of the Bragg peaks, and a low $c$ drives them apart, while a high $c$ drives them together. Combined with the choice of material, these five parameters are all that are needed for describing the multilayer.

For a fixed set of parameters, increasing $N$ will increase reflectivity due to a more continuous distribution of Bragg peaks, up to a point where the absorption becomes dominant and outweighs the benefit of adding layers. The $\Gamma$ parameter has a more complex interaction, but in general increasing $\Gamma$ results in an absorption at high energies as the amount of high-Z material is increased. The minimum d-spacing of the stack, $d_{\text {min }}$ is through the Bragg law related to the maximum energy that can be reflected at a specific incidence angle through

$$
d_{\min } \propto \frac{1}{E_{\max } \theta_{\max }} .
$$

This parameter is important for the off-axis response, since as can be seen, maintaining reflectivity at $E_{\text {max }}$ when $\theta_{\max }$ increases due to off-axis rays, requires $d_{\min }$ to decrease. Thus in general $d_{\min }$ controls the high energy 
response. Conversely $d_{\max }$ controls the low energy reflectivity through a similar relation

$$
d_{\max } \propto \frac{1}{E_{\min } \theta_{\min }}
$$

Increasing $d_{\text {max }}$ beyond the point where $\theta_{\min }<\theta_{c}$ only serves to add absorption to the stack, and therefore in general $d_{\max }\left(\theta_{\min }=\theta_{c}\right)$. Of course for both $d_{\min }$ and $d_{\max }$ there are more complex interactions which create features that add and subtract to the response at different energies, and the above relations are used as guidelines.

The exact reflectivity from a multilayer stack can be calculated using Parratt's recursive formula, ${ }^{9}$ but inverting the formula to find the best parameters in an analytical way is not a trivial matter. Instead we have developed a Figure of Merit, FOM, code to help find the right combination. The next sections detail our method and results.

\section{BASELINE DESIGN}

The baseline design is summarized in Table 1. The total number of shells in one optic is 130 , and to make the optimization more manageable we subdivide the shells into 10 mirror groups, where group 1 contains the mirrors with the smallest radii and group 10 the largest. The chosen material combinations are $\mathrm{Pt} / \mathrm{SiC}$ and $\mathrm{W} / \mathrm{Si}$. $\mathrm{Pt} / \mathrm{SiC}$ provides response up to the $\mathrm{Pt} \mathrm{K}$-edge at $78.4 \mathrm{keV}$, while $\mathrm{W}$ provides high response at intermediate energies, but only works up to $69.4 \mathrm{keV}$. SiC and Si provides very smooth interfaces and experimentally it has been found that the average rms roughness for $\sigma_{P t / S i C}=0.45 \mathrm{~nm}$ and for $\sigma_{W / S i}=0.43 \mathrm{~nm} .{ }^{10}$

The optical constants for $\mathrm{Pt}, \mathrm{W}, \mathrm{SiC}$ and $\mathrm{Si}$ have been theoretically calculated using the database of the National Institute of Standards and Technology, and been experimentally verified. ${ }^{11}$

Fabrication constraints on the thickness of the $\mathrm{Pt}$, layer places a limit on $d_{\min }$ and $\mathrm{\Gamma}$, as it is not possible to make this layer any thinner than $0.8 \mathrm{~nm}$ without compromising the roughness. Further more the accuracy with which $\Gamma$ can be deposited is estimated to bc \pm 0.05 . This places a lower constraint on $d_{\min }=2.5 \mathrm{~nm}$ and for all designs we fix $\Gamma \equiv 0.38$ to be safe beyond the point where the roughness degrades.

Schedule is a driving factor and due to a slow deposit time of $\mathrm{SiC}$, we have limited the total thickness of the stack to 1 micron.

Table 1. Baseline

\begin{tabular}{|c|c|}
\hline Parameter & Value \\
\hline \hline Minimum Radius & $5.44 \mathrm{~cm}$ \\
Maximum Radius & $19.12 \mathrm{~cm}$ \\
Focal Length & $1015 \mathrm{~cm}$ \\
Number of Shells & 130 \\
Number of Groups & 10 \\
High density materials & $\mathrm{Pt}$ and W \\
Low density materials & $\mathrm{SiC}$ and Si \\
\hline Stack constraints & \\
\hline Minimum $d_{\min }$ & $2.5 \mathrm{~nm}$ \\
$\Gamma$ & 0.38 \\
$\sigma_{\mathrm{Pt} / \mathrm{SiC}}$ & $0.45 \mathrm{~nm}$ \\
$\sigma_{\mathrm{W} / \mathrm{Si}}$ & $0.43 \mathrm{~nm}$ \\
Maximum $\mathrm{D}_{\text {stack }}$ & $1 \mathrm{micron}$ \\
\hline
\end{tabular}

\subsection{Top layer}

As can be seen from Figure 1 left panel the critical angle of $\mathrm{Pt}$ and SiC, as a function of grazing incidence angle, passes through the energy range of the optic, and for all mirror groups, energies below $20 \mathrm{keV}$ are in the regime of total cxternal reflection. However, total external reflection is only possible if the strata is actually thick enough, ${ }^{5}$ 
and as shown in Figure 1 right panel, at $20 \mathrm{keV}$ the 1/e penetration depth for mirror group 1 is $\sim 3 \mathrm{~nm}$ and increases to $\sim 34 \mathrm{~nm}$ for mirror group 10. Should another interface appear within this depth, the ray is no longer totally externally reflected but interacts with the stack below. For the innermost shell the penetration depth at $55 \mathrm{keV}$ is $\sim 30 \mathrm{~nm}$, while $d_{\max }$ for a $30 \mathrm{keV}$ reflection, which is the SiC critical energy for the smallest graze angle, is $\sim 15 \mathrm{~nm}$. With $\Gamma=0.38$ this is a Pt layer thickness of $5.7 \mathrm{~nm}$ and much less than the $1 / \mathrm{e}$ penetration depth.

There are two ways of dealing with this problem. One way is to increase $d_{\text {max }}$ of the power-law stack by a factor of 2 to what Eqn (4) requires, which results in a thicker stack. Another way is to change the top bi-layer directly to be different from the rest of the power-law stack. Both inethods work well, but with slightly different energy responses. Because of our constraints on fabrication, we found that changing the top bi-layer directly is a better method for NuSTAR.

For fabrication reasons we require the final layer to be a $\mathrm{SiC}$ or Si sealing coating, but instead of having it be $62 \%$ of the layer thickness, we can simply change the value of $\Gamma$ at the top layer to be higher. This results in a thin $\mathrm{SiC}$ or Si layer and thick $\mathrm{Pt}$ or W. Since the amplitude reflectivity decays exponential as a function of the penetration depth, a $\Gamma_{\text {top }}$ in the range of $0.7-0.8$ is sufficient to ensure most of the wave is reflected.

We will in the following distinguish between the top and stack fractional thickness as $\Gamma_{\text {top }}$ and $\Gamma_{\text {stack }}$.
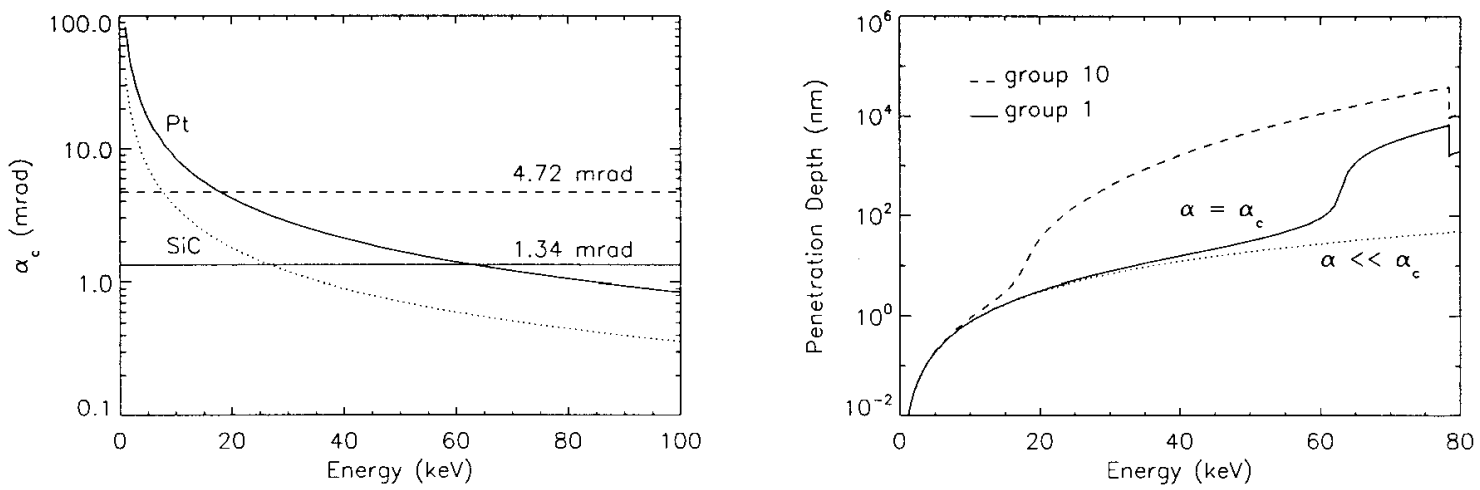

Figure 1. LEFT: The critical angle of $\mathrm{Pt}$ and $\mathrm{SiC}$ is shown as a function of energy. The horizontal solid line represents the smallest graze angle of the optic, and the dashed line largest graze angle. All energies left of the intersections are totally externally reflected, and so for the innermost shells energies up to $60 \mathrm{keV}$ are in the regime of total external reflection, while for the outermost mirror group it is $20 \mathrm{keV}$. RIGHT: The penetration depth of $\mathrm{Pt}$, where the amplitude reflectivity has dropped with 1/e, as a function of energy is plotted for 3 cases. The dotted curve is independent of absorption and holds true when the angles are $\alpha_{i n c}<<\alpha_{c}$. The solid curve is the penetration depth at the critical angle for $\alpha_{i n c}=1.34$ mrad, and the dashed curve for $\alpha_{i n c}=4.72 \mathrm{mrad}$. At $60 \mathrm{keV}$ the depth is $60 \mathrm{~nm}$ and so despite being in the regime of total external reflection, parts of the wave will penetrate into the multilayer stack below and exhibit characteristics of the stack.

\section{SIMULATIONS}

The Figure of Merit method of optimizing multilayers was first developed and used for HEFT ${ }^{12}$ but is entirely general for grazing incidence optics. It uses a function which is the weighted energy integral of the field-of-view averaged effective area for each mirror shell, summed over all mirrors

$$
\mathrm{FOM}=\frac{\sum_{i=1}^{N} \int_{E_{\min }}^{E_{\max }} \mathrm{d} E A_{i}(E) W_{E}(E)}{\left(E_{\max }-E_{\min }\right)\left\langle W_{E}\right\rangle}
$$

The value of the FOM is in $\mathrm{cm}^{2}$ and is the energy averaged effective area of a mirror group. 


\subsection{1 $A_{i}(E)$, Effective Area}

The on-axis effective area of a Wolter-I is given by the product of the projected collecting area and the reflectivity of the mirrors. The area of the i'th shell at radius $r_{i}$ with half-opening angle $\alpha_{i}$ and mirror length $l$, is thus

$$
A_{i}(E)=2 \pi r_{i} \alpha_{i} l \times\left[R\left(E, \alpha_{i}\right)\right]^{2} .
$$

$R\left(E, \alpha_{i}\right)$ is a matrix of reflectivity as a function of energies and angles, and it is calculated using the Névot-Croce formalism ${ }^{13}$ and Parratt's recursive formula. ${ }^{9}$

For an off-axis photon the incidence angle on the secondary mirror is different than the primary, and so in order to correctly calculate the effective area an incidence angle distribution of the two mirrors is needed. If the photon arrives at off-axis angle $\psi$ the incidence angles on the primary and secondary mirrors are $\theta_{1}=\alpha+\psi$ and $\theta_{2}=\alpha-\psi$. The change in effective area as a function of off-axis angle is contained in an angular weighting function, $W_{\text {inc }}\left(\alpha_{i}, \psi\right)$ which is generated by uniform illumination of the optic aperture and ray-tracing of the rays through the optic. With this weighting function the effective area becomes

$$
A_{i}(E)=\int_{-\psi}^{\psi} W_{i n c}\left(\alpha_{i}, \psi\right) 2 \pi r_{i} \alpha_{i} l\left[R\left(E, \alpha_{i}+\psi\right) R\left(E, \alpha_{i}-\psi\right)\right] d \psi
$$

\subsection{2 $W_{E}$, Energy Weighting Function}

The energy weighting function sets the desired spectral response. $\left\langle W_{E}\right\rangle$ is the average value of the weighting response over the energy range $E_{\min }-E_{\max }$, and including it in the denominator of Eqn (5) ensures that, the Figure of Merit is independent of the normalization of $W_{E}$. The primary function of the energy weighting is to ensure that the low energy effective area does not dominate the FOM. Through a series of tests we found that a linear function provides a suitable balance between low and high energy effective area, and that using weighting functions of higher power does not improve the spectral response.

\subsection{Optimization technique}

Given the power-law parameterizations of the bi-layer thickness distribution from Eqn (2) the number of parameters necessary to define our graded multilayer with top layer is six. For values of power-law index $c$ and number of bi-layers $\mathrm{N}$, the constants $a$ and $b$ from the power law can be uniquely determined by $d_{\min }$ and $d_{\max }$. The fifth and sixth parameters are the high density material fractional thickness $\Gamma_{\text {stack }}$ and $\Gamma_{\text {top }}$.

With $d_{\min }, \Gamma_{\text {stack }}$ and $\Gamma_{\text {top }}$ fixed, the total thickness of one layer is determined by the total number of bi-layers $\mathrm{N}$, and only weakly dependent on the choice of $c$ and $d_{\max }$. More bi-layers will increase the reflectivity until at some point absorption starts to dominate in the stack and no more reflectivity is gained from adding more layers. The optimization is thus run by choosing a value of $\mathrm{N}$ that empirically is known to be less than optimum. The values of $d_{\max }$ and $c$ are then optimized until the FOM peaks, after which $\mathrm{N}$ is incremented by $25 \%$ and the process is repeated until the FOM decreases following successive values of $\mathrm{N}$.

\section{RESULTS}

Table 2 summarizes the results of our optimizations. Mirror groups 1 through 7 are coated with $\mathrm{Pt} / \mathrm{SiC}$ and have $\Gamma_{\text {stack }}=0.38, \Gamma_{\text {Top }}=0.7$ and $\sigma_{P t / S i C}=0.45 \mathrm{~nm}$. Mirror groups 8 through 10 are coated with W/Si and have $\Gamma_{\text {stack }}=0.38, \Gamma_{\text {Top }}=0.8$ and $\sigma_{W / S i}=0.43 \mathrm{~nm}$. Column two lists the minimum and maximum graze angles of the mirror group, and column three the shells belonging to the mirror group. It, should be noted that there are 130 actively reflective shells, but the table includes 133 since 3 shells, shells 66-68, are replaced with a structural intermediate mandrel. Columns ' $\mathrm{D}_{\max }$ ', ' $\mathrm{N}$ ' and ' $\mathrm{c}$ ' are the FOM optimized parameters, and the actual FOM is in the final column.

Figure 2 illustrates the FOM process for mirror group 2. Left panel shows the Figure of Merit curve as a function of $\mathrm{N}$. At each data point the listed number is the total thickness of the stack, $D_{\text {stack. For each value }}$ of $\mathrm{N}$ the design is optimized, and the optimized reflectivity curves for $\mathrm{N}=62,96,150$ and 233 are shown in the right panel. It can be observed how the reflectivity progressively gets better, but only change very little at the top of the FOM curve. This allows us to pick designs that are a little further down the curve, like $\mathrm{N}=150$ which in terms of the FOM only has an cumulative difference in energy averaged effective area for that mirror group of $0.05 \mathrm{~cm}^{2}$. 


\begin{tabular}{|c|c|c|c||c|c|c||c|c|c|c|c|}
\hline $\begin{array}{c}\text { Group } \\
\mathrm{Pt} / \mathrm{SiC}\end{array}$ & $\begin{array}{c}\text { Angle } \\
{[\mathrm{mrad}]}\end{array}$ & $\begin{array}{c}\text { Layer } \\
\text { range }\end{array}$ & $\begin{array}{c}\mathrm{D}_{\min } \\
{[\mathrm{nm}]}\end{array}$ & $\begin{array}{c}\mathrm{D}_{\max } \\
{[\mathrm{nm}]}\end{array}$ & $\mathrm{N}$ & $\mathrm{c}$ & $\begin{array}{c}\sigma_{P t / S i C} \\
{[\mathrm{~nm}]}\end{array}$ & $\Gamma_{\text {Top }}$ & $\Gamma_{\text {stack }}$ & $\begin{array}{c}\mathrm{D}_{\text {stack }} \\
{[\text { micron }]}\end{array}$ & $\begin{array}{c}\text { FOM } \\
{\left[\mathrm{cm}^{2}\right]}\end{array}$ \\
\hline 1 & $1.34-1.52$ & $1-12$ & 3.17 & 12.812 & 150 & 0.250 & 0.45 & 0.7 & 0.38 & 0.631 & 5.934 \\
2 & -1.73 & $13-24$ & 3.05 & 12.093 & 150 & 0.256 & 0.45 & 0.7 & 0.38 & 0.611 & 7.77 \\
3 & -1.96 & $25-36$ & 2.93 & 11.656 & 187 & 0.238 & 0.45 & 0.7 & 0.38 & 0.716 & 9.029 \\
4 & -2.22 & $37-49$ & 2.81 & 11.906 & 233 & 0.213 & 0.45 & 0.7 & 0.38 & 0.833 & 10.739 \\
5 & -2.52 & $50-62$ & 2.5 & 11.031 & 291 & 0.238 & 0.45 & 0.7 & 0.38 & 0.952 & 11.237 \\
6 & -2.85 & $63-76$ & 2.5 & 10.898 & 291 & 0.229 & 0.45 & 0.7 & 0.38 & 0.942 & 10.929 \\
7 & -3.23 & $77-89$ & 2.5 & 10.384 & 291 & 0.217 & 0.45 & 0.7 & 0.38 & 0.929 & 10.599 \\
\hline \hline Group & Angle & Layer & $\mathrm{D}_{\min }$ & $\mathrm{D}_{\max }$ & $\mathrm{N}$ & $\mathrm{c}$ & $\sigma_{W / \text { Si }}$ & $\Gamma_{\text {Top }}$ & $\Gamma_{\text {stack }}^{2}$ & $\mathrm{D}_{\text {stack }}$ & $\mathrm{FOM}$ \\
W/Si & {$[\mathrm{mrad}]$} & range & {$[\mathrm{nm}]$} & {$[\mathrm{nm}]$} & & & {$[\mathrm{nm}]$} & & & {$[$ micron] } & {$\left[\mathrm{cm}^{2}\right]$} \\
\hline 8 & -3.67 & $90-104$ & 2.5 & 9.522 & 291 & 0.238 & 0.43 & 0.8 & 0.38 & 0.955 & 11.695 \\
9 & -4.16 & $105-118$ & 2.5 & 8.394 & 291 & 0.220 & 0.43 & 0.8 & 0.38 & 0.934 & 9.342 \\
10 & -4.72 & $119-133$ & 2.5 & 7.447 & 291 & 0.190 & 0.43 & 0.8 & 0.38 & 0.902 & 8.154 \\
\hline
\end{tabular}

Table 2. Optimized NuSTAR design. Groups $1-7$ are coated with Pt/SiC and groups $8-10$ are coated with W/Si. Columns 'D $D_{\text {max }}$ ', 'N' and 'c' are the optimized parameters.
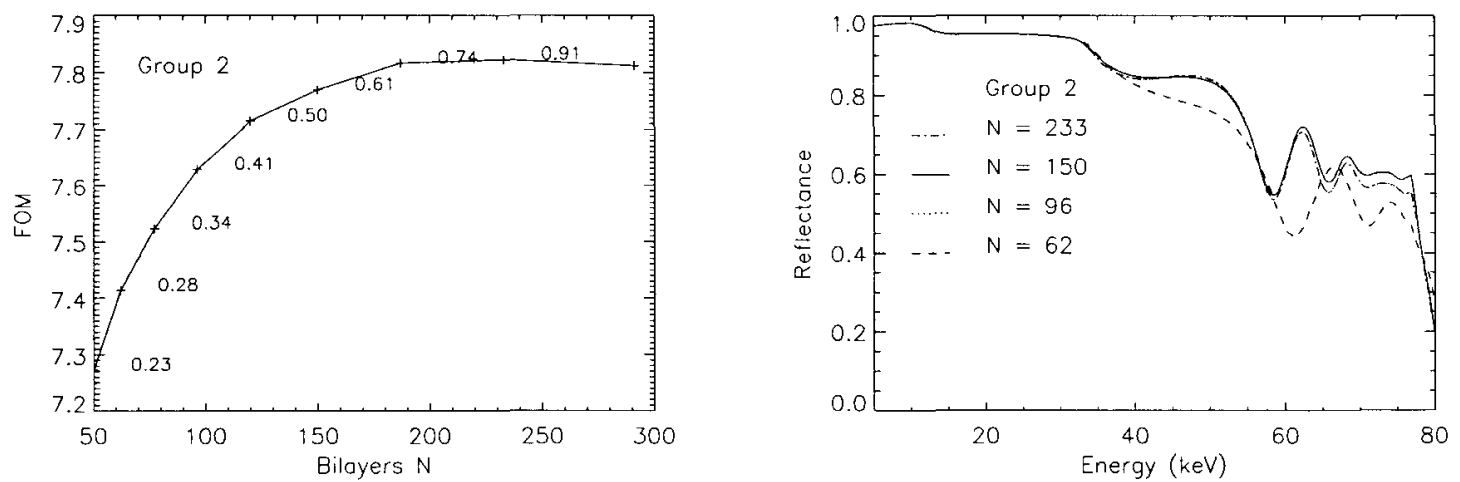

Figure 2. LEFT: Figure of Merit curve as a function of bi-layer number N. The curve peaks at $N=233$ where the total thickness is $D_{\text {stack }}=0.91$ microns. However, to meet the thickness requirement we chose $\mathrm{N}=150$ where $D_{\text {stack }}=0.61$ micron, and as shown in the right panel the difference in reflectance is small. RIGHT: Reflectance curves for $\mathrm{N}=150,96$ and 62 at an incidence angle of $\theta_{i n c}=1.7 \mathrm{mrad}$. As can be seen the difference in reflectance for $\mathrm{N}=233$ and $\mathrm{N}=150$ is small, while the difference in thickness is a reduction of $33 \%$. 


\subsection{Total thickness of the stack and $d_{\min }$}

Counter intuitively perhaps, $d_{\text {min }}$ along with $\mathrm{N}$, controls the thickness of the stack. The total thickness of the stack is a very important factor, as deposit times for $\mathrm{SiC}$ are very slow and the fabrication schedule is very tight.

Table 3 shows the values of $d_{\text {min }}$ as calculated using Eqn (3) for two different angles and energy $78 \mathrm{keV}: \theta_{\text {inc }}$ and $\theta_{i n c}+6$ arcmin, where $\theta_{i n c}$ is the maximum graze angle of the mirror group. The final column shows the final choice of $d_{\min }$ used in the design. From the first two columns it can be seen how dramatically $d_{m i n}$ changes through the groups, and that the thickness decreases as the graze angle increases.

To understand how $d_{\min }$ impacts the reflectivity and $\mathrm{N}$, to the right is a plot of the reflectivity curve of mirror group 1 at an angle $\theta=1.52 \mathrm{mrad}+4 \operatorname{arcmin}$, optimized for three different $d_{\min }=2.5 \mathrm{~nm}, 3.1 \mathrm{~nm}$ and $4.1 \mathrm{~nm}$. Respectively the optimum number of bi-layers, $\mathrm{N}$, were found to be 291,150 and 77 , that is $\mathrm{N}$ decreases with increasing $d_{\text {min }}$. However, as can be seen from the plot, the reflectivity due to the Bragg law, falls of faster for off-axis angles with increasing $d_{\text {min }}$. At 2 arcmin off-axis angle the cutoff in the $d_{\min }=4.1$ nm curve starts moving away from $78 \mathrm{keV}$, and as can be seen in the plot, at 4 arcmin the same is happening to the $d_{\min }=3.1$ nm curve. The choice of $d_{m i n}$ is thus a tradeoff in layer thickness and off-axis performance.

\begin{tabular}{|c|c|c|c|}
\hline Group & $\begin{array}{c}\mathrm{d}_{\min }\left(\theta_{\text {inc }}\right) \\
{[\mathrm{nm}]}\end{array}$ & $\begin{array}{c}\mathrm{d}_{\min }\left(\theta_{\text {inc }}+6^{\prime}\right) \\
{[\mathrm{nm}]}\end{array}$ & $\begin{array}{c}\text { Design } \mathrm{d}_{\min } \\
{[\mathrm{nm}]}\end{array}$ \\
\hline \hline 1 & 5.22 & 2.39 & 3.17 \\
2 & 4.61 & 2.25 & 3.05 \\
3 & 4.06 & 2.11 & 2.93 \\
4 & 3.58 & 1.98 & 2.81 \\
5 & 3.16 & 1.84 & 2.5 \\
6 & 2.78 & 1.79 & 2.5 \\
7 & 2.45 & 1.57 & 2.5 \\
8 & 2.16 & 1.45 & 2.5 \\
9 & 1.91 & 1.33 & 2.5 \\
10 & 1.68 & 1.21 & 2.5 \\
\hline
\end{tabular}

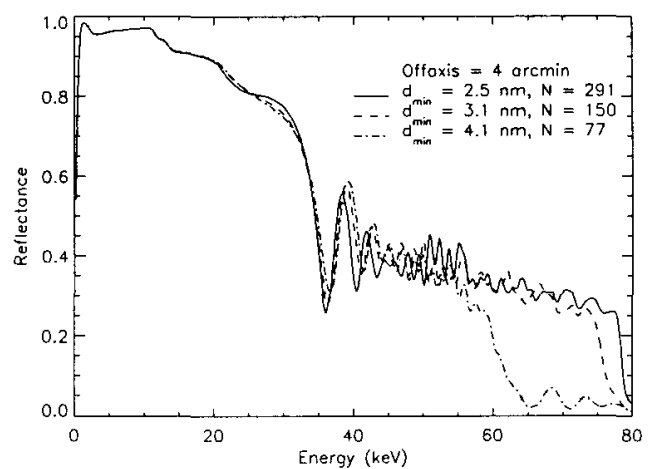

Table 3. LEFT: The table shows the value of $\mathrm{d}_{\text {min }}$ as calculated using the Bragg law for various angles. The second column shows the minimum d-spacing necessary to reflect a $78 \mathrm{keV}$ photon at $\theta_{i n c}=$ maximum graze angle in the mirror group. The third column shows the minimum d-spacing necessary for a reflection of a $78 \mathrm{keV}$ photon at $\theta_{\text {inc }}+6$ arcmin. The forth column shows the chosen minimum d-spacings. RIGHT: The Figure shows for mirror group 1 at a graze angle of $1.52 \mathrm{mrad}+4$ arcmin for different values of $\mathrm{d}_{\min }$.

\subsection{Toplayer}

To demonstrate the importance of designing the top bi-layer Figure 3 left panel shows mirror group 2 with $\Gamma_{\text {top }}=0.7$ and $\Gamma_{\text {top }}=\Gamma_{\text {stack }}$ (dashed). For the case $\Gamma_{\text {top }}=\Gamma_{\text {stack }}$ the first dip at $\sim 35 \mathrm{keV}$ is the critical angle of $\mathrm{Pt} / \mathrm{SiC}$ bi-layer, followed by the oscillations of the Bragg peaks. For the solid curve the top bi-layer is $\Gamma=0.7$ resulting in the extension of the critical angle to the location of the first Bragg peak, and eliminating the deep minima of the dashed curve. This same effect could have been achieved with doubling the maximum $d$-spacing, but at the cost of a significantly thicker stack.

The choice of $\Gamma_{\text {top }}$ has been found through simulations. Increasing the thickness of $\mathrm{Pt}$ will flatten the spectrum at 30 to $60 \mathrm{keV}$ and remove the features which are due to interactions with the multilayer below. However, when $\Gamma_{t o p}$ continues to increase the minima between the critical energy and the location of the first maxima deepens and broadens, while energies above the critical energy starts getting absorbed due to the increasing Pt thickness. A $\Gamma \equiv 0.7$ was found to be a good balance between these effects.

For W/Si a value of $\Gamma=0.8$ was found to be more appropriate as shown in Figure 3 right panel. The plot shows the fraction of the effective area for mirror groups 8-10 coated with W/Si with different $\Gamma_{\text {top }}$ as a fraction, for the same mirror groups optimized for $\mathrm{Pt} / \mathrm{SiC}$ with $\Gamma=0.7$. The gain in effective area going from $\mathrm{Pt} / \mathrm{SiC}$ to $\mathrm{W} / \mathrm{Si}$ for the outer mirror groups is on average $20 \%$ in the 30 to $70 \mathrm{keV}$ range. There is a loss at 10 to $25 \mathrm{keV}$, mainly due to the smaller critical angle of $\mathrm{W}$, and increasing $\Gamma_{\text {top }}$ partially remedies the situation. Increasing 
$\Gamma_{\text {top }}$ beyond 0.8 , while improving the area at 10 to $25 \mathrm{keV}$, it unfortunately also creates a dip at $\sim 28 \mathrm{keV}$. To balance this we chose a $\Gamma_{\text {top }} \equiv 0.8$
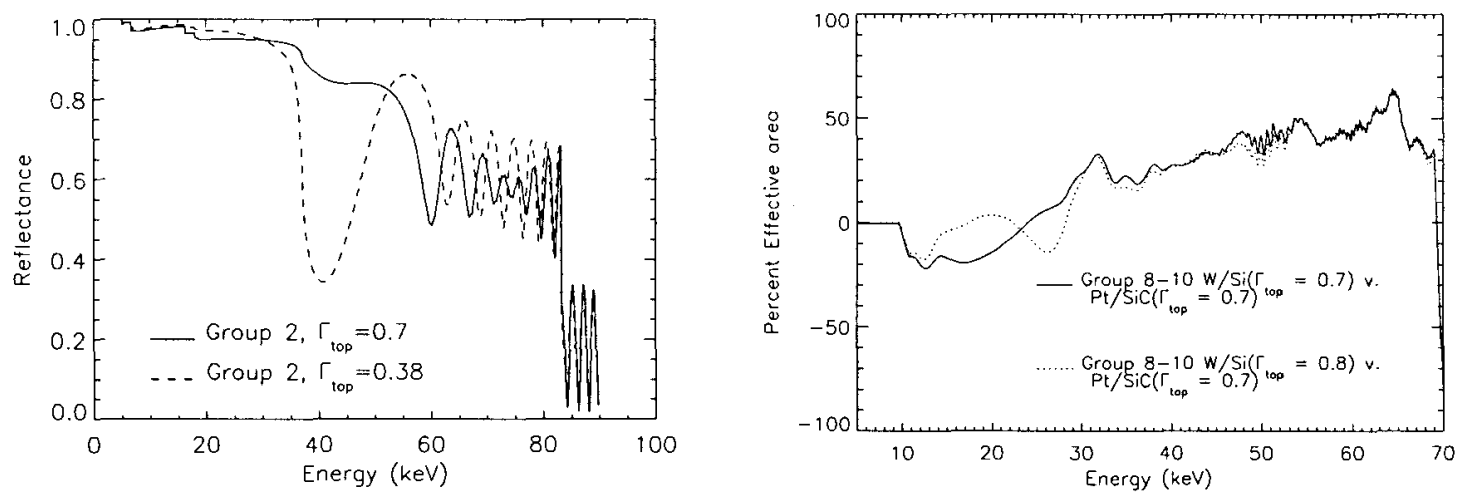

Figure 3. LEFT: Reflectance curve of mirror group 2 at grazing incidence angle $1.8 \mathrm{mrad}$. The solid curve has $\Gamma_{t o p}=0.7$, and the dashed curve has $\Gamma_{t o p}=0.38$. Here it can be clearly seen how the thick Pt layer extends the total reflection up to $\sim 55 \mathrm{keV}$, at the cost of a few absorption features at around $20 \mathrm{keV}$. RIGHT: The fractional on axis effective area of groups 8-10 for a W/Si( $\left.\Gamma_{t o p}=0.7\right)$ and $\mathrm{W} / \mathrm{Si}\left(\Gamma_{t o p}=0.8\right)$ as compared to $\mathrm{Pt} / \mathrm{SiC}\left(\Gamma_{t o p}=0.7\right)$. The gain in effective area in the energy range $30-70 \mathrm{keV}$ by using $\mathrm{W} / \mathrm{Si}$ can clearly be seen, but it comes at the cost of a decrease in the $10-30$ $\mathrm{keV}$ range. This can in part be remedied by using $\Gamma_{t o p}=0.8$ for $\mathrm{W} / \mathrm{Si}$.

\subsection{Measurements}

Figure 4 and 5 show measurements of a Pt/SiC and W/Si coating. In Figure 4 on the left is a measurement of mirror group 2, coated with $\mathrm{Pt} / \mathrm{SiC}$, taken at $8 \mathrm{keV}$ at the National Space Institute, DTU-space, Denmark. The coating was deposited on an actual flight substrate of a curvature consistent with mirror group 2 . The solid curve is the data, and the crosses the model of mirror group 2. From 10 to 40 mrad the model fit is very good, while beyond $40 \mathrm{mrad}$ statistics become poor and the fit noisy. The importance of measurements at high angles is to constrain the roughness, which at $8 \mathrm{keV}$ was found to be $\sigma=0.4 \mathrm{~nm}$. The left panel shows a different Pt/SiC coating for mirror group 3 at $50 \mathrm{keV}$ taken at the Brookhaven National Laboratory. The cut-off at 4.5 mrad is due to the choice of $d_{\text {min }}$.

Figure 5 shows a measurements of mirror group 8 , coated with W/Si, taken at $8 \mathrm{kcV}$ and $50 \mathrm{keV}$. This recipe has been coated on a flat $\mathrm{Si}$ Wafer with substrate rms roughness $\sigma=0.4 \mathrm{~nm}$. The solid line is the actual measurement and the crosses the model. In the left panel the fit up to an angle of $20 \mathrm{mrad}$ is very good. Above $20 \mathrm{mrad}$ the data is less frequently sampled and most features are lost. In the right panel the same coating is shown at $50 \mathrm{keV}$. A misalignment of the critical angle at $\sim 2 \mathrm{mrad}$ can be observed which is due to a possible variation in $\Gamma_{\text {stack }}$ and $\Gamma_{\text {top }}$. The cut-off at $\sim 6 \mathrm{mrad}$ is due to the value of $d_{\min }$.

All measurements show good agreement, with the recipe model and the predicted rm roughness, proving that the recipes are viable and reproducible.

\subsection{Effective Area}

From a science perspective the exact details of each mirror is less important than the over all effective area of the optic. The effective area of two optics is shown in Figure 6 from on-axis to 9 arc-minutes off-axis. The effective area has been derived using a ray-trace on a precise model of the optics, including effects such as the obstruction from graphite spacers, missing glass pieces between segments, aperture clipping, and the effect of scattering from the mirrors with an energy and angle independent HPD $=40$ arc-seconds. The effective area both on and off-axis is a smooth function with few prominent features except for the bump at $70 \mathrm{keV}$ where the $\mathrm{W} / \mathrm{Si}$ transitions to $\mathrm{Pt} / \mathrm{SiC}$. The decrease in off-axis area is roughly linear with off-axis angle. 

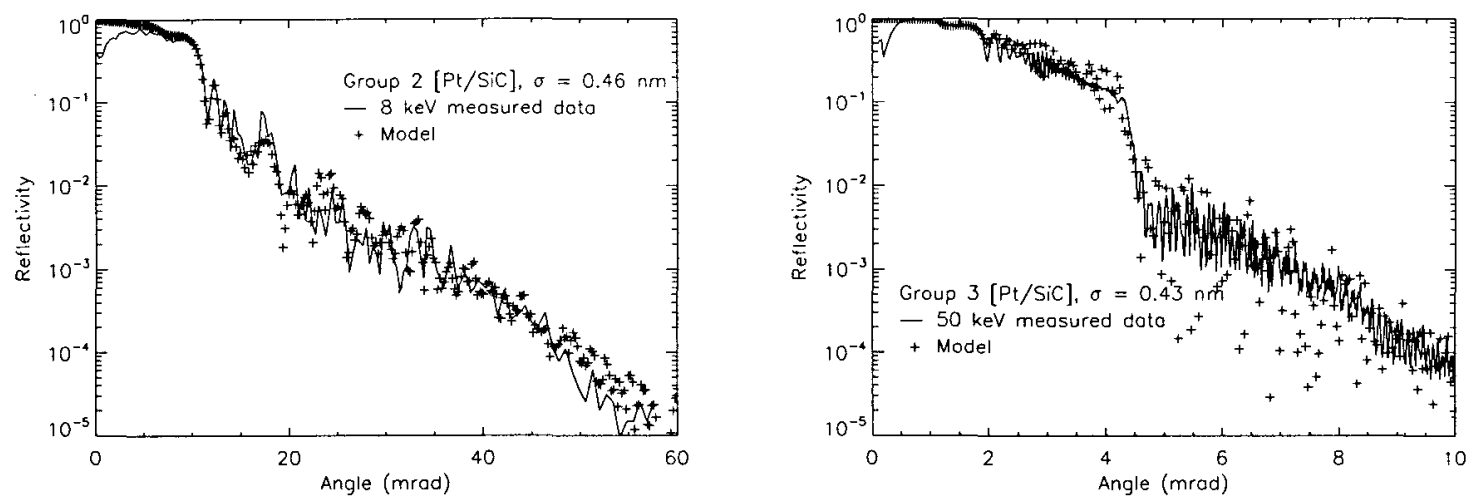

Figure 4. LEFT: Pt/SiC group 2 flight coating at $8 \mathrm{kcV}$. RIGHT: Pt/SiC group 3 flight coating at $50 \mathrm{keV}$ taken at Brookhaven National Laboratory.
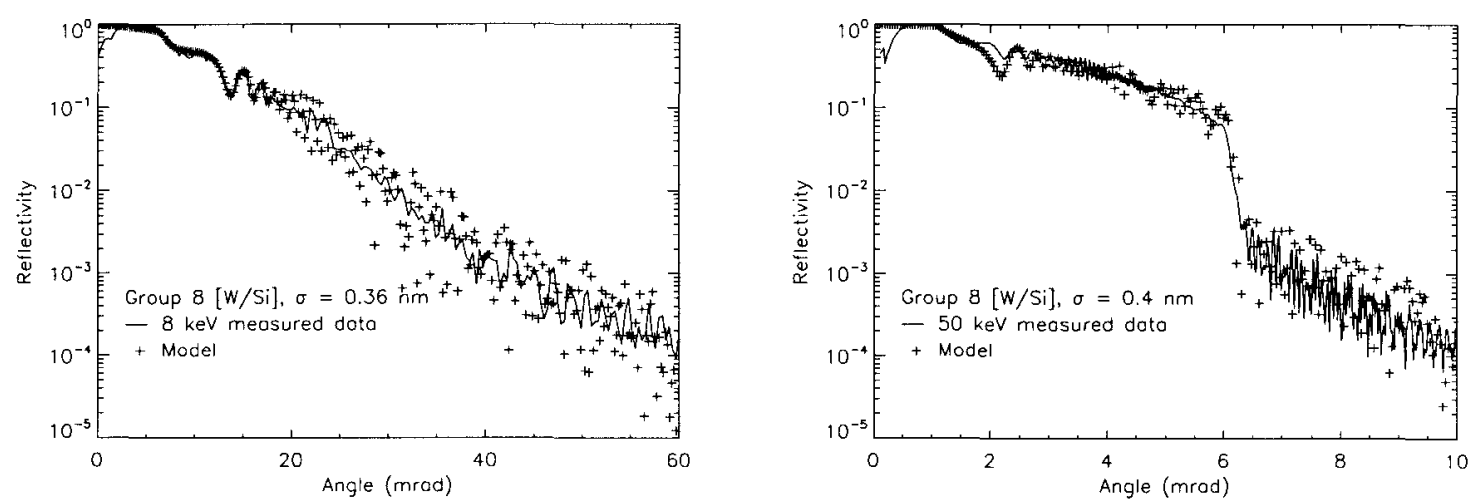

Figure 5. LEFT: W/Si group 8 flight coating at $8 \mathrm{keV}$. RIGHT: W/Si group 8 flight coating at $50 \mathrm{keV}$ taken at Brookhaven National Laboratory. 


\section{CONCLUSION}

We have optimized the NuSTAR multilayer recipes using a Figure of Merit process, and the result are the 10 mirror recipes listed in Table 2. The goal was to achieve a smooth effective area with high off-axis area, and we have done this with a combination of depth graded multilayers, and manipulating the top layer to control the placement of the critical angle. We have produced test coatings of a few of these recipes and verified that they were viable and corresponded to the models.

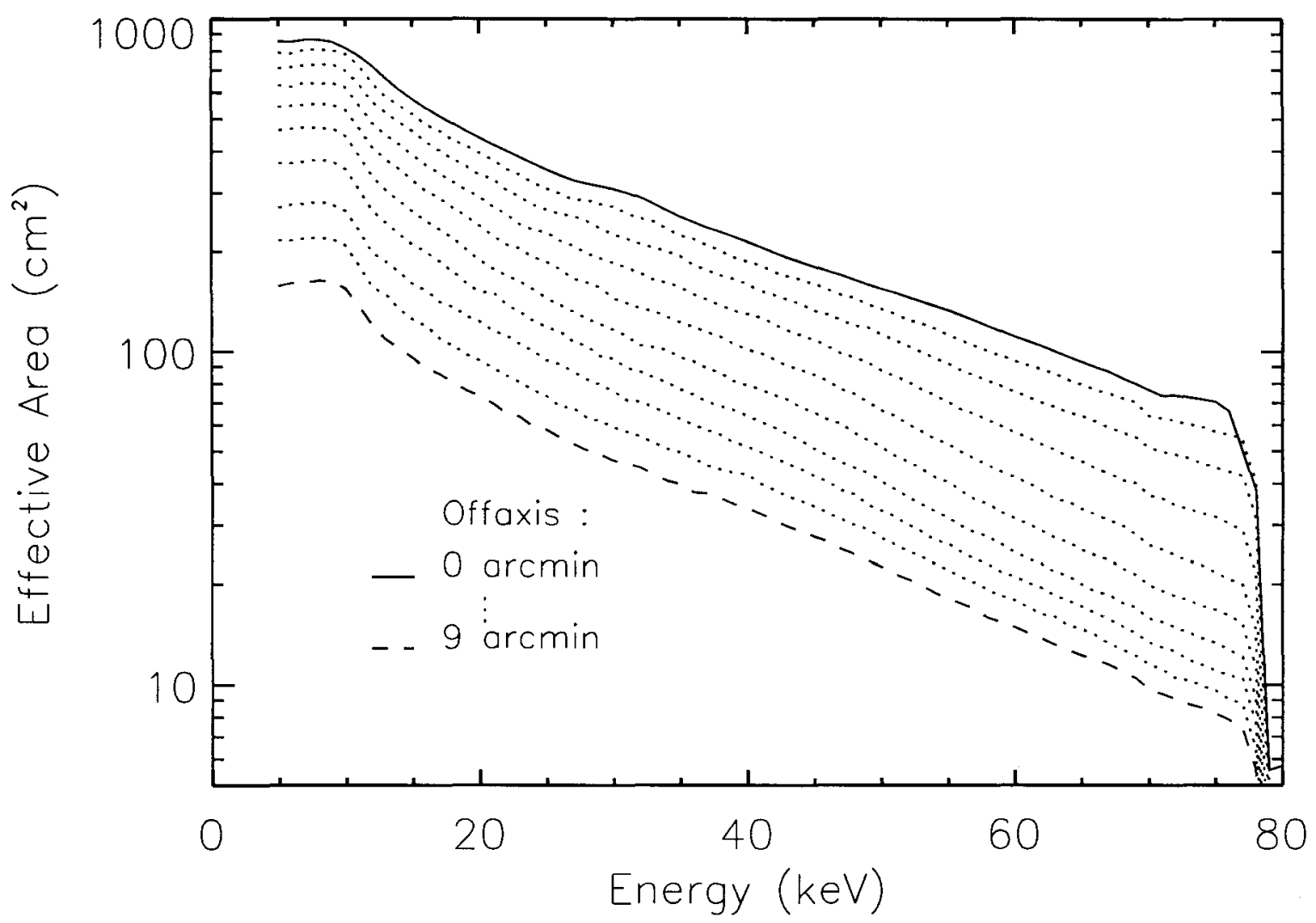

Figure 6. Effective area of two optics for the design summarized in Table 2. The off-axis effective area has been raytraced and includes all known obscuration effects from missing glass, graphite spacers, aperture clipping at the focal plane and also includes scattering of mirrors with a HPD $=40$ arc-seconds.

\section{REFERENCES}

[1] Schwartz, D. and et al. Proc. SPIE 4012, 28-40 (2000).

[2] Jansen, F. and et al. A $8 A$ 365, L1-L6 (2001).

[3] Giacconi, R. and et al., [The Einstein Observatory and future x-ray telescopes], Palo Alto, Annual Reviews, Inc. (1981).

[4] Wolter, H. Annalen der Physik 10, 94 (1952).

[5] Born, M. and Wolf, E., [Principles of Optics], Oxford.

[6] Harrison, F. A. and et al Experimental Astronomy 20, 131-137 (2005).

[7] Jensen, C. P. and et al Proc. SPIE 4851, 724-733 (2003). 
[8] Joensen, K. and et al Appl Opt. 34, 7935 (1995).

[9] Parratt, L. G. Revue Phys 95, 359 (1954).

[10] Madsen, K. K. and et al Proc. SPIE 4851, 724 (2003).

[11] Jensen, C. P. and et al Proc. SPIE 7011, 7011K-1 - 7011K-9 (2008).

[12] Mao, P. H. and et al Appl Opt. 38, 4766-4775 (1999).

[13] Nevot, L. and Corce, P. Revue Phys 15, 761 (1980). 